\title{
Contracepção pós-abortamento: relação com a atenção em contracepção e intenção reprodutiva
}

\author{
Post-abortion contraception: effects of \\ contraception services and reproductive intention
}

\author{
Anticoncepción pos-aborto: relación entre \\ la atención en anticoncepción e intención \\ reproductiva
}

\section{Resumo}

Aconselhamento em contracepção e oferta de insumos contraceptivos são parte da atenção pós-abortamento e influenciam positivamente o uso de métodos contraceptivos. Estudos que mostram tais evidências são realizados, predominantemente, em países sem restrições legais ao aborto e com adequada atenção às mulheres que interrompem a gravidez. No entanto, pouco se sabe a respeito das práticas contraceptivas em contextos de ilegalidade do aborto, caso do Brasil, em que a atenção em contracepção pós-abortamento é inadequada. O objetivo foi analisar o efeito da atenção em contracepção no uso de preservativo masculino, anticonceptivo oral

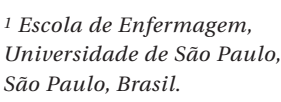

1 Escola de Enfermagem, Universidade de São Paulo, São Paulo, Brasil. e injetável nos seis meses pós-abortamento, considerando a intenção reprodutiva. Os resultados mostraram que a atenção em contracepção tem efeito positivo apenas sobre o uso do anticonceptivo oral nos seis meses pós-abortamento, desde que a mulher tenha passado por consulta médica no mesmo mês em que recebeu orientações sobre contracepção. Uma ou outra ação não tem efeito significativo quando ocorre isoladamente.

Aborto; Anticoncepção; Planejamento Familiar;

Saúde Sexual e Reprodutiva 


\section{Introdução}

O aconselhamento em contracepção e a oferta de insumos contraceptivos ainda durante a hospitalização são parte da atenção pós-abortamento e têm mostrado efeito positivo na adoção de métodos contraceptivos (MAC) 1, especialmente os reversíveis de longa duração, como DIU e implantes 2. Estudos que mostram tais evidências são realizados, predominantemente, em países sem restrições legais ao aborto e, portanto, com atenção adequada às mulheres que interrompem sua gravidez. Ainda, a maior parte desses estudos é do tipo ensaio aleatorizado, em que um protocolo de aconselhamento contraceptivo é seguido rigorosamente para avaliar seu efeito no uso de MAC e na ocorrência de novos abortamentos 3 . No entanto, pouco se sabe a respeito das práticas contraceptivas em contextos de restrições legais ao aborto, como é o caso do Brasil. Embora o Ministério da Saúde tenha reeditado o manual de atenção humanizada ao abortamento em 2011 com o intuito de subsidiar o atendimento integral e de qualidade às mulheres em situação de abortamento ${ }^{1}$ - induzido ou espontâneo - e cumprir os planos de ação das Conferências Internacionais de Cairo e Pequim, bem como das Metas do Desenvolvimento do Milênio, a atenção em contracepção pós-abortamento durante a hospitalização no país ainda é inadequada 4 .

Além da atenção em contracepção, que consiste na orientação sobre MAC disponíveis no país e na oferta imediata de MAC escolhidos pela mulher/casal 1, a intenção reprodutiva 2,5 também tem sido considerada como elemento importante no processo de tomada de decisão quanto ao uso de MAC. Isso porque as mulheres com abortamento espontâneo podem querer engravidar tão logo seja possível, ao passo que as mulheres com abortamento induzido podem estar mais motivadas a evitar uma gravidez.

Diante desse quadro, nossa pergunta de pesquisa é: a chance de usar preservativo masculino, pílula oral e hormonal injetável difere nos seis meses pós-abortamento segundo a atenção em contracepção e a intenção reprodutiva? Para responder a ela, foi analisada a relação entre a atenção em contracepção, a intenção reprodutiva e o uso de pílula oral, preservativo masculino e hormonal injetável nos seis meses posteriores à vivência de um abortamento. A hipótese é que a atenção em contracepção - da forma como é ofertada atualmente no país - não exerce efeito no uso de MAC, mas a intenção reprodutiva sim.

\section{Métodos}

Trata-se de um estudo observacional longitudinal conduzido com amostra não probabilística de mulheres hospitalizadas por abortamento em maternidade pública da cidade de São Paulo, Brasil, entre agosto e dezembro de 2011. Todas as mulheres nessa condição foram convidadas a participar do estudo ( $n=187)$, mas houve recusas $(\mathrm{n}=17)$. As 170 mulheres que aceitaram participar foram entrevistadas ainda durante a hospitalização, quando responderam a um questionário estruturado sobre suas características sociodemográficas e reprodutivas. A etiologia do abortamento não foi questionada, pelo fato de que haveria subnotificação de abortos provocados. As entrevistas mensais por telefone foram realizadas durante seis meses após a alta hospitalar. Esse período foi definido porque a Organização Mundial da Saúde (OMS) preconiza que mulheres que passam por abortamento devem esperar seis meses para engravidar novamente. As questões versaram sobre o uso de MAC no mês anterior, sobre a atenção em contracepção recebida e sobre a intenção de engravidar novamente.

No primeiro mês pós-abortamento, $147 \mathrm{mu}$ lheres foram localizadas. No sexto mês, foram entrevistadas 105 mulheres e contabilizadas 25 perdas de seguimento, por conta de números de telefone inexistentes. Mulheres que relataram gravidez confirmada foram excluídas do estudo $(\mathrm{n}=17)$. Este estudo contempla, pois, 696 observações pessoa/mês.

As variáveis dependentes foram: (a) uso de preservativo masculino; (b) uso de anticoncepcional oral; e (c) uso de anticoncepcional injetável nos seis meses pós-abortamento. Os demais tipos de MAC, como DIU e tradicionais, não foram analisados por terem sido pouco relatados. As principais variáveis explicativas foram a atenção em contracepção e a intenção reproduti$v a$. A priori, a atenção em contracepção durante a hospitalização não foi analisada neste estudo pelo fato de que poucas mulheres referiram ter tido alta hospitalar com MAC prescrito. A variável atenção em contracepção foi construída com base no fato de que, no âmbito da atenção básica do Sistema Único de Saúde (SUS), as mulheres/ casais recebem orientação sobre MAC de diversos profissionais de saúde, individualmente ou em grupo, porém a aquisição de MAC hormonais de uso regular só ocorre, em geral, por meio de prescrição médica, fornecida durante consulta médica. Assim, as mulheres que relataram não ter recebido orientação sobre contracepção nem passado por consulta médica no mês anterior foram agrupadas na categoria nenhuma; as mu- 
lheres que relataram ter recebido algum tipo de orientação sobre contracepção foram agrupadas na categoria apenas orientação; as mulheres que relataram ter passado por consulta médica no mês anterior foram agrupadas na categoria apenas consulta médica; e, por fim, as mulheres que relataram ter recebido algum tipo de orientação sobre contracepção e passado por consulta médica no mesmo mês foram agrupadas na categoria ambas. Já a variável intenção reprodutiva foi construída com a pergunta "Quando gostaria de engravidar novamente?”, feita mensalmente a cada mulher. As mulheres foram agrupadas em: não quer mais engravidar, quer engravidar novamente e ainda não pensou sobre isso. As variáveis de ajuste foram: a idade (anos), a escolaridade (anos), o planejamento da gravidez que terminou em abortamento (não planejada, ambivalente e planejada), paridade (número de filhos tidos) e tempo após o abortamento (meses).

Por se tratarem de medidas repetidas e, por isto, observações dependentes, a análise dos dados foi conduzida por intermédio de modelos lineares generalizados mistos para dados longitudinais, tendo em conta os efeitos aleatórios na estimação dos parâmetros, no Stata 13.0 (StataCorp LP, College Station, Estados Unidos). Foi conduzido um modelo para cada tipo de MAC, e as variáveis independentes foram inseridas simultaneamente.

Este estudo foi aprovado por Comitê de Ética em Pesquisa da instituição hospitalar em que foi realizado. Foram obtidos Termos de Consentimento Livre e Esclarecido de todas as mulheres que aceitaram participar do estudo.

\section{Resultados}

As mulheres entrevistadas no primeiro mês pósabortamento tinham 29,1 anos de idade (DP = $7,4$ ) e 9,3 anos de estudo ( $\mathrm{DP}=2,5)$, em média. Três quartos estavam unidas $(76,9 \%) ; 68 \%$ já tinham filhos e $66,7 \%$ gostariam de ter mais filhos. A gravidez que terminou em abortamento não foi planejada para $21,1 \%$ das mulheres; $29,9 \%$ relataram gravidez planejada e $49 \%$ eram ambivalentes.

A Tabela 1 mostra que a chance de uma mulher usar o preservativo masculino, o anticonceptivo oral e o injetável diferiu ao longo dos seis meses pós-abortamento (OR ajustado $=0,85$; 1,49 e 1,58, respectivamente). No primeiro mês, $40 \%$ das mulheres relataram ter usado o preservativo masculino, proporção bem maior do que as que relataram ter usado o anticonceptivo oral ou injetável. Tal proporção diminuiu no período, chegando a ser um pouco mais baixa que o uso do anticonceptivo oral. A proporção de uso de injetável praticamente triplicou.

A atenção em contracepção foi estatisticamente associada apenas ao uso do anticonceptivo oral (Tabela 1). Nota-se que esse efeito só foi significativo quando a orientação sobre contracepção ocorreu no mesmo mês que a consulta médica. $\mathrm{O}$ relato de um ou outro evento isoladamente não teve qualquer efeito sobre o uso do anticonceptivo oral. Tampouco foi observado qualquer efeito da intenção reprodutiva sobre o uso dos MAC investigados. Especificamente em relação ao preservativo masculino, a cada aumento de um ano de estudo na escolaridade da mulher, foi observada 1,26 vez a chance de usálo. No que concerne ao uso do injetável, a idade foi a única variável estatisticamente significativa (OR ajustado $=0,81)$.

\section{Discussão}

Os resultados mostram que a atenção em contracepção nos seis meses pós-abortamento não influenciou o uso de preservativo masculino nem de injetável nesse período. Sua influência só foi encontrada no uso do anticonceptivo oral quando a consulta médica ocorreu no mesmo mês que as orientações sobre contracepção, indicando que ações que englobam simultaneamente orientação e oferta de insumos são capazes de promover o uso de MAC pós-abortamento 6 .

Foi também verificado que as mulheres usaram métodos reversíveis de curta duração, que são o preservativo masculino e o anticonceptivo oral, coincidentemente MAC em que as descontinuidades ocorrem com maior frequência, podendo acarretar mais chance de ocorrência de gestações não planejadas 1,2 . Por certo, a razão pela qual esses MAC foram comumente usados pode estar relacionada ao pouco incentivo ou acesso a outros tipos de MAC. Nesse sentido, há fortes evidências de que as mulheres tendem a escolher MAC mais eficazes e de longa duração após o abortamento, como o DIU 2,6, fato que não foi constatado neste estudo. O uso do injetável não foi frequente, embora tenha aumentado no período. O mix contraceptivo centrado nesses três MAC foi também observado na Pesquisa Nacional de Demografia e Saúde (PNDS 2006) para as mulheres em geral 7. O efeito não significativo da intenção reprodutiva no uso de MAC reforça que pode ter sido o acesso o elemento preponderante na decisão sobre o uso. De toda forma, para a OMS 8, International Federation of Gynecology and Obstetrics (FIGO) ${ }^{9}$ e o próprio Ministério da Saúde 1 , o cuidado integral à saúde da mulher em situação de abortamento deve incluir, além do 
Proporção de uso e razão de chances de uso de preservativo masculino, anticonceptivo oral e injetável seis meses após o abortamento. São Paulo, Brasil, 2011-2012.

\begin{tabular}{|c|c|c|c|}
\hline & \multicolumn{3}{|c|}{ Método contraceptivo } \\
\hline & Preservativo masculino & Anticonceptivo oral & Anticonceptivo injetável \\
\hline & $\%$ & $\%$ & $\%$ \\
\hline \multicolumn{4}{|l|}{ Meses após o abortamento } \\
\hline 1 & 40,0 & 15,2 & 4,1 \\
\hline 2 & 39,1 & 31,9 & 7,9 \\
\hline 3 & 39,2 & 33,8 & 10,0 \\
\hline 4 & 32,8 & 36,9 & 9,0 \\
\hline 5 & 33,3 & 35,9 & 7,9 \\
\hline \multirow[t]{2}{*}{6} & 30,8 & 33,6 & 12,1 \\
\hline & OR ajustado (IC95\%) & OR ajustado (IC95\%) & OR ajustadao (IC95\%) \\
\hline \multicolumn{4}{|l|}{ Atenção em contracepção } \\
\hline Nenhuma & 1,00 & 1,00 & 1,00 \\
\hline Apenas orientação & $0,80(0,30-2,16)$ & $1,34(0,40-4,48)$ & $0,39(0,02-9,20)$ \\
\hline Apenas consulta médica & $1,26(0,70-2,26)$ & $0,70(0,33-1,48)$ & $1,44(0,39-5,40)$ \\
\hline Ambas & $1,05(0,54-2,04)$ & $3,83(1,64-8,96)$ & $1,40(0,36-5,40)$ \\
\hline \multicolumn{4}{|l|}{ Intenção reprodutiva } \\
\hline Não quer mais engravidar & 1,00 & 1,00 & 1,00 \\
\hline Quer engravidar novamente & $1,01(0,41-2,49)$ & $1,30(0,41-4,07)$ & $0,78(0,15-3,97)$ \\
\hline Não pensou sobre isto & $1,09(0,44-2,74)$ & $1,50(0,48-4,67)$ & $0,74(0,14-3,86)$ \\
\hline \multicolumn{4}{|l|}{ Planejamento da gravidez } \\
\hline Gravidez não planejada & 1,00 & 1,00 & 1,00 \\
\hline Gravidez ambivalente & $0,55(0,18-1,63)$ & $1,22(0,22-6,89)$ & $0,39(0,05-2,90)$ \\
\hline Gravidez planejada & $0,71(0,21-2,42)$ & $1,18(0,17-8,32)$ & $0,08(0,01-1,44)$ \\
\hline Idade & $1,05(0,98-1,12)$ & $1,01(0,90-1,12)$ & $0,81(0,68-0,96)$ \\
\hline Escolaridade & $1,26(1,04-1,52)$ & $1,04(0,77-1,40)$ & $0,69(0,47-1,00)$ \\
\hline Paridade & $1,06(0,67-1,68)$ & $0,61(0,29-1,29)$ & $2,76(0,98-7,78)$ \\
\hline Meses após o abortamento & $0,85(0,75-0,96)$ & $1,49(1,27-1,76)$ & $1,58(1,18-2,12)$ \\
\hline
\end{tabular}

IC95\%: intervalo de 95\% de confiança; OR: odds ratio.

tratamento de emergência, o acesso universal ao planejamento reprodutivo, inclusive orientações para mulheres que desejam uma nova gestação possam engravidar em condições clínicas apropriadas para o desenvolvimento saudável da gravidez. Isso é possível por meio do fortalecimento das redes de cuidado em todos os níveis de atenção, assim como a capacitação dos trabalhadores para a efetiva implementação dos protocolos já disponíveis. Esse conjunto de ações pode ampliar o acesso das mulheres no período pós-abortamento ao aconselhamento em contracepção e MAC, de forma a garantir seus direitos sexuais e reprodutivos, bem como contemplar suas intenções reprodutivas e diretrizes clínicas claramente estabelecidas no manual 1 . 


\section{Agradecimentos}

À FAPESP pelo apoio financeiro (processo 2010/ 15297-0).

\section{Referências}

1. Área Técnica de Saúde da Mulher, Departamento de Ações Programáticas Estratégicas, Secretaria de Atenção à Saúde, Ministério da Saúde. Atenção humanizada ao abortamento: norma técnica. Brasília: Ministério da Saúde; 2005. (Série A. Normas e Manuais Técnicos). (Série Direitos Sexuais e Direitos Reprodutivos. Caderno, 4).

2. Madden T, Secura GM, Allsworth JE, Peipert JF. Comparison of contraceptive method chosen by women with and without a recent history of induced abortion. Contraception 2011; 84:571-7.

3. Schunmann C, Glasier A. Specialist contraceptive counseling and provision after termination of pregnancy improves uptake of long-acting methods but does not prevent repeat abortion: a randomized trial. Hum Reprod 2006; 21:2296-303.

4. Aquino EML, Menezes G, Barreto-de-Araújo TV, Alves MT, Alves SV, Almeida MCC, et al. Qualidade da atenção ao aborto no Sistema Único de Saúde do Nordeste brasileiro: o que dizem as mulheres? Ciênc Saúde Coletiva 2012; 17:1765-76.
5. Schünmann C, Glasier A. Measuring pregnancy intention and its relationship with contraceptive use among women undergoing therapeutic abortion. Contraception 2006; 73:520-4.

6. Ceylan A, Ertem M, Saka G, Akdeniz N. Post abortion family planning counseling as a tool to increase contraception use. BMC Public Health 2009; 9:20.

7. Ministério da Saúde. Pesquisa Nacional de Demografia e Saúde 2006: relatório. Brasília: Ministério da Saúde; 2008.

8. Organização Mundial da Saúde. Abortamento seguro: orientação técnica e de políticas para sistemas de saúde. 2aa Ed. Brasília: Organização Mundial da Saúde; 2013.

9. International Federation of Gynecology and Obstetrics. Consensus statement - family planning: a key component of post abortion care. London: International Federation of Gynecology and Obstetrics; 2009 


\section{Abstract}

Contraceptive counseling and the supply of contraceptive methods are part of post-abortion care and positively influence the subsequent use of contraceptive methods. Studies showing such evidence have been conducted predominantly in countries with no legal restrictions on abortion and with adequate care for women that terminate a pregnancy. However, little is known about contraceptive practices in contexts where abortion is illegal, as in Brazil, in which post-abortion contraceptive care is inadequate. The objective of this study was to analyze the effect of contraceptive care on male condom use and oral and injectable contraceptives in the six months post-abortion, considering reproductive intention. The results showed that contraceptive care only has a positive effect on the use of oral contraceptives in the first six months post-abortion, as long as the woman had a medical consultation in the same month in which she received information on contraception. One or the other intervention alone had no significant impact.

Abortion; Contraception; Family Planning (Public Health); Sexual and Reproductive Heatlh

\section{Resumen}

El asesoramiento en anticoncepción y la oferta de insumos anticonceptivos forma parte de la atención posaborto e influencian positivamente el uso de métodos anticonceptivos. Existen estudios que muestran que tales evidencias se realizan, predominantemente, en países sin restricciones legales al aborto y con una adecuada atención a las mujeres que interrumpen su embarazo. No obstante, poco se sabe respecto a las prácticas anticonceptivas en contextos de ilegalidad del aborto, como en el caso de Brasil, donde la atención en anticoncepción pos-aborto es inadecuada. El objetivo fue analizar el efecto de la atención en anticoncepción en el uso del preservativo masculino, anticonceptivo oral e inyectable en los seis meses pos-aborto, considerando la intención reproductiva. Los resultados mostraron que la atención en anticoncepción tiene un efecto positivo sólo sobre el uso del anticonceptivo oral tras los seis meses pos-aborto, siempre que la mujer haya pasado por consulta médica durante el mismo mes en que recibió orientaciones sobre anticoncepción. Una u otra acción no tienen efectos significativos cuando se producen aisladamente.

Aborto; Anticoncepción; Planificación Familiar; Salud Sexual y Reproductiva
Recebido em 26/Jun/2015

Versão final reapresentada em 27/Out/2015 Aprovado em 01/Dez/2015 